\title{
Lack of MECP2 gene transcription on the duplicated alleles of two MECP2 duplication females with opposite skewed X chromosome inactivation
}

\author{
Yixi Sun ${ }^{1}$, Yali Yang ${ }^{1}$, Yuqin Luo ${ }^{1}$, Min Chen ${ }^{1}$, Liya Wang ${ }^{1}$, Yingzhi Huang ${ }^{1}$, Yanmei \\ Yang $^{1}$, and Mingyue Dong ${ }^{1}$ \\ ${ }^{1}$ Zhejiang University
}

April 5, 2021

\begin{abstract}
Xq28 (involving MECP2) duplication syndrome is a severe neurodevelopmental disorder in males, most females are asymptomatic carriers, but there are phenotypic heterogeneities in the females. Skewed X-chromosome inactivation (XCI) seems to prevent duplicated region activation in asymptomatic females, but it remains controversial. Herein we reported two asymptomatic females (daughter and mother) with interstitial Xq28 duplication. HUMARA and RP2 assays showed that both had complete skewed XCI, the Xq28 duplicated chromosome was inactivated in the daughter, but surprisingly, it was activated in her mother. Interestingly, by combining RNA sequencing and whole-exome sequencing, we confirmed that XIST only expressed in the Xq28 duplication chromosomes of the two females, indicating that the Xq28 duplication chromosomes were inactive. Meanwhile, MECP2 and most XCI genes in the duplicated X-chromosomes were not transcriptionally expressed or upregulated, precluding major clinical phenotypes in the two females, especially the mother. We showed that XCI status detected by RNA sequencing was more relevant for establishing the clinical phenotype of MECP2 duplication females. It suggested there were other factors maintaining the XCI status in addition to DNA methylation, a possible additional inhibition mechanism occured at the transcriptional level in the unmethylated X-chromosome, counter balancing the MECP2 duplication's detrimental phenotype effects
\end{abstract}

Lack of $M E C P 2$ gene transcription on the duplicated alleles of two $M E C P 2$ duplication females with opposite skewed $\mathrm{X}$ chromosome inactivation

Yixi Sun ${ }^{1,2,3}$, Yali Yang ${ }^{1,2,3}$, Yuqin Luo ${ }^{1,2,3}$, Min Chen ${ }^{1,2,3}$, Liya Wang ${ }^{1,2,3}$, Yingzhi Huang ${ }^{1,2,3}$, Yanmei Yang ${ }^{1,2,3}$, Minyue Dong ${ }^{1,2,3, *}$

1. Department of Reproductive Genetics, Women's Hospital, School of Medicine, Zhejiang University, Hangzhou, Zhejiang, 310006, China.

2. Key Laboratory of Reproductive Genetics, Ministry of Education (Zhejiang University), Hangzhou, Zhejiang, 310006, China.

3. Key Laboratory of Women's Reproductive Health of Zhejiang Province, Hangzhou, Zhejiang, 310006, China.

\section{*Correspondence:}

Minyue Dong, MD/PhD, Department of Reproductive Genetics, Women's Hospital, School of Medicine, Zhejiang University, Hangzhou, Zhejiang, 310006, China.

Tel: +86-571-89991860, E-mail:dongmy@zju.edu.cn 


\begin{abstract}
Xq28 (involving MECP2 ) duplication syndrome is a severe neurodevelopmental disorder in males, most females are asymptomatic carriers, but there are phenotypic heterogeneities in the females. Skewed Xchromosome inactivation (XCI) seems to prevent duplicated region activation in asymptomatic females, but it remains controversial. Herein we reported two asymptomatic females (daughter and mother) with interstitial Xq28 duplication. HUMARA and RP2 assays showed that both had complete skewed XCI, the Xq28 duplicated chromosome was inactivated in the daughter, but surprisingly, it was activated in her mother. Interestingly, by combining RNA sequencing and whole-exome sequencing, we confirmed that $X I S T$ only expressed in the Xq28 duplication chromosomes of the two females, indicating that the Xq28 duplication chromosomes were inactive. Meanwhile, MECP2 and most XCI genes in the duplicated Xchromosomes were not transcriptionally expressed or upregulated, precluding major clinical phenotypes in the two females, especially the mother. We showed that XCI status detected by RNA sequencing was more relevant for establishing the clinical phenotype of $M E C P 2$ duplication females. It suggested there were other factors maintaining the XCI status in addition to DNA methylation, a possible additional inhibition mechanism occured at the transcriptional level in the unmethylated X-chromosome, counter balancing the $M E C P 2$ duplication's detrimental phenotype effects.
\end{abstract}

\title{
KEYWORDS
}

MECP2 duplication, skewed X-chromosome inactivation (XCI), human androgen receptor (HUMARA) assay, RNA sequencing, allele-specific expression (ASE)

\section{INTRODUCTION}

Xq28 (involving MECP2) duplication syndrome (MDS) is a severe neurodevelopmental disorder. Signs and symptoms include infantile hypotonia, delayed psychomotor development, severe intellectual disability, poor speech development, progressive spasticity, recurrent respiratory infections, and seizures (Van Esch, 1993). MDS is caused by a duplication (or triplication) of the gene methyl CpG binding protein 2 (MECP2 ), which increases the expression of transcription regulator MeCP2 (Makrythanasis et al., 2010; Ramocki et al., 2009). MeCP2 protein overexpression could have detrimental effects on brain development and function, as shown in mouse models and humans (Collins et al., 2004; Ramocki \& Zoghbi, 2008; Van Esch et al., 2005).

Initially, MDS was considered fully penetrant in males, and most females with duplications were asymptomatic carriers. However, later reports revealed a mild to severe clinical phenotype in more than 20 affected females with $M E C P 2$ duplication, including larger cytogenetic changes resulting in $M E C P 2$ duplication and intrachromosomal (intraC) Xq28 duplication (El Chehadeh et al., 2017; Van Esch, 1993).

$\mathrm{X}$-chromosome inactivation (XCI) is a transcriptional silencing mechanism of the X-chromosome initiated by the transcription of XIST, which encodes a long non-coding RNA, transcribes mono-allelically, and spreads in cis along the inactive $\mathrm{X}(\mathrm{Xi})$ chromosome. $\mathrm{XCI}$ induces progressive epigenetic silencing by recruiting chromatin remodeling enzymatic complexes, thereby imposing repressive histone and DNA changes to the $\mathrm{Xi}$ chromosome. One $\mathrm{X}$-chromosome is inactivated at random in females, but some females show preferential inactivation for one of the two X-chromosomes (skewed XCI) (Lee, 2011). Skewed XCI can cause phenotypic heterogeneity of X-linked disorders in females (Horga et al., 2019; Orstavik, 2009; Y. Sun et al., 2019). XCI seems to be essential for MECP2 expression in Xq28 duplication females, which affects the phenotype.

XCI pattern analysis has generally been conducted by human androgen receptor (HUMARA) assay, a polymerase chain reaction (PCR)-based X-chromosome inactivation assay that uses a methylation-sensitive restriction enzyme. However, using the classic mean, some reports did not correlate XCI and the severity of the phenotype in females affected by Xq28 duplication (Bijlsma et al., 2012; El Chehadeh et al., 2017; Fieremans et al., 2014; Scott Schwoerer et al., 2014). We speculated it was because the HUMARA assay did not directly reflect the transcriptional level of the genes in Xq28. Recently, quantification of allelic read counts and allele-specific expression (ASE) by RNA sequencing has been used as a direct XCI assay (Tukiainen et al., 2017; Zito et al., 2019). We investigated two females in a daughter-mother relationship, 
each possessing interstitial Xq28 duplication (involving $M E C P 2$ ) opposite skewed XCI at the methylated level, as detected by HUMARA assay and RP 2 assay. Through RNA sequencing (RNA-seq), we revealed that chromosomes with $\mathrm{Xq} 28$ duplication were both silent at the transcriptional level, and the dosages of genes $M E C P 2$ and IRAK1 spanning the Xq28-duplicated region were not upregulated. This finding explains why both females were asymptomatic, especially the mother.

\section{METHODS AND PATIENTS}

\section{Patients}

A 34-year-old healthy woman II2 had given birth to a boy with typical symptoms of MECP2 duplication syndrome. These symptoms included infantile hypotonia, delayed psychomotor development, poor speech development, intellectual disability, and recurrent respiratory infections. She wanted to give birth to a second child. To explore the genetic cause and phenotypic effects of skewed XCI, we performed a single-nucleotide polymorphism (SNP) array and whole-exome sequencing (WES) for her and her asymptomatic mother I2. Meanwhile, we detected the XCI states of the two females by HUMARA / RP2 assays and RNA-seq.

This study followed the Ethics Committee of Women's Hospital's recommendations, School of Medicine at Zhejiang University. All participants provided informed consent in accordance with the Declaration of Helsinki. The Review Board of the Women's Hospital, School of Medicine, Zhejiang University in China approved the study protocol.

\section{DNA/RNA extraction and qPCR}

We extracted genomic DNA samples of peripheral blood and fetal amniotic fluid with the GentraPuregene Kit (Qiagen, Germany). We extracted total RNA with TRIzol reagent according to the manufacturer's instructions (Invitrogen). Quantitative real-time (qRT-PCR) was carried out using SYBR Green PCR Master Mix (Takara, Japan) on the Applied Biosystems 7900HT system. Supp. Table S1 lists the primers. Melting curve analyses confirmed that all primers were specific for their respective transcript. We used the $\Delta \Delta \mathrm{Ct}$ method to determine relative $\mathrm{DNA} / \mathrm{cDNA}$ levels and determined the fold change by the value of $2^{-\Delta \Delta^{\prime \prime} \tau}$.

\section{SNP array}

We performed the SNP array using the CytoScan HD Array Kit (Affymetrix, USA) according to the manufacturer's instruction, with around 2,600,000 markers, including 750,000 SNP probes and 1,900,000 nonpolymorphism probes used for comprehensive whole-genome coverage. Data were analyzed by Chromosome Analysis Suite software (Affymetrix, Santa Clara, CA) based on GRCh38 assembly.

\section{XCI analysis}

$\mathrm{XCI}$ in the Xq28-duplication females was analyzed by PCR amplification of the HUMARA gene and retinitis pigmentosa (RPQ ) locus, aspreviously described (Allen, Zoghbi, Moseley, Rosenblatt, \& Belmont, 1992; Machado et al., 2014).

\section{RNA-Seq}

We performed RNA-Seq by Biomarker Technologies (Beijing, China) and generated sequencing libraries using NEBNextR Ultra Directional RNA Library Prep Kit for IlluminaR (NEB, USA) following the manufacturer's recommendations. Clustering of the index-coded samples was performed on a cBot Cluster Generation System using TruSeq PE Cluster Kit v4-cBot-HS (Illumina) according to the manufacturer's instructions. After cluster generation, the library preparations were sequenced on an Illumina HiSeq Xten platform. Raw data (raw reads) in the FASTQ format were first processed through in-house Perl scripts, and clean data (clean reads) were obtained by removing reads containing an adapter, reads containing ploy-N, and lowquality reads from raw data. Then, the clean reads were mapped to the reference genome sequence. HISAT2 tools software was used for reference genome mapping. We used Picard-tools v1.41 and SAMtools v0.1.18 to sort through reads, remove duplicated reads, and merge each sample's bam alignment results. GATK2 or 
SAMtools software was used to perform SNP calling. Raw VCF files were filtered using the GATK standard filter method, and only SNPs with a distance $>5$ were retained. Differential expression analysis of two groups was performed using the DESeq R package (1.10.1)

\section{Whole-Exome Sequencing (WES)}

WES was performed by Biomarker Technologies (Beijing, China). The sequencing libraries were generated using the NimbleGen SeqCap EZ Human Exome V3 (Roche, Basel, Swiss) following the manufacturer's recommendations. Clustering of the index-coded samples was performed on a cBot Cluster Generation System (Illumina, USA) according to the manufacturer's instructions. After cluster generation, the library preparations were sequenced on an Illumina HiSeq X Ten platform with a $150 \mathrm{bp}$ paired-end module.

Burrows-Wheeler Aligner v0.7.13-r1126 was used to align each sample's clean reads with the reference genome using default parameters. Alignment files were converted to BAM files using SAMtools software. Variant calling was performed for all samples by using the Haplotype Caller in GATK software.

\section{Fluorescence in situ hybridization}

Fluorescence in situ hybridization (FISH) was performed in the female and her mother. Xq28 was tagged with BAC RP11-119A22 (Illumina) labeled in the red spectrum. The centromere probe was Vysis CEP X (DXZ1), which was labeled in the green spectrum (Abbott Laboratories).

\section{Results}

1. Xq28 intrachromosomal duplications were identified in female II2 (daughter) and I2 (mother) by SNP array, qPCR, and FISH analyses

We detected I1, I2, and II2 by Affymetrix CytoScan HD Array. It showed a $437 \mathrm{~kb}$ duplication on the Xq28 region (chrX:153858452-154332213 in the genome build GRCh38) and $284 \mathrm{~kb}$ duplication on the Xq27.3 region (chrX:144988272-145271978: in the genome build GRCh38) in female II2 and her mother I2 (Figure 1B), which demonstrated that the Xq28 and Xq27.3 duplications were maternally inherited. The Xq28 region contains 13 Online Mendelian Inheritance in Man (OMIM) genes (including MECP2, IRAK1, $L 1 C A M, N A A 10$, and $H C F C 1$ ). We also performed qPCR to verify the results, and one copy of the gene dosages of MECP2 and IRAK1 were detected in male I1, whereas two copies were detected in the normal control female, and three copies were detected in I2 and II2 (Figures 1C, D). FISH analysis on metaphase chromosomes of females I2 and II2 revealed signals for the MECP2 -containing probe RP11-119A22 only at Xq28, which indicates that the Xq28 duplications were tandem repeats that had not integrated elsewhere in the genome (Figures 1E, F).

\section{HUMARA and RP2 assays showed that females I2 and II2 possessed complete skewed $\mathrm{XCI}$, but in the opposite X-chromosome}

As presented in Figure 2, we performed PCR-based HUMARA and RP2 assays to assess the XCI patterns in I2, II2. After digestion with the methylation-sensitive restriction enzyme HpaII, we found that only the inactive X-chromosome could synthesize the PCR products. We determined the origin of the inactivate X-chromosome by segregation analysis. The undigested PCR product of II2 gave two peaks $(267 / 287$ in HUMARA and 359/381 in RP2 ). A single peak represented a single peak (287 in HUMARA and 359 in $R P 2$ ) for the HpaII-digested product (Figures 2C, F), which indicated that the inactivate allele was inherited from her mother I2. The assay of the undigested PCR product of the mother I2 gave two peaks (281/287 in HUMARA and 359/374 in RP2 ). A major peak (281 inHUMARA and 374 in RP2 ), detected by assaying the HpaII digested product (Figures 2B, E), was different from that in the inactivated product allele of II2. The above results indicate that complete skewing of XCI was found in the female II2 and her mother I2, and was different from that of the inactivate X-chromosome (Figures 2, 4). The 287 bp peak of $H U M A R A$ PCR products, 359 bp peak of $R P 2$ PCR products in II2, and the $\mathrm{Xq} 28$ duplication were derived from the mother I2, which were linked to the same X-chromosome. However, the allele, which gave a peak of 
287 bp after HUMARA PCR, a peak of 359 bp after RP2 PCR, and the Xq28 duplication, was inactivated in II2 but activated in I2.

3. No additional serious pathogenic mutations on the X-chromosomes were detected in I2 by exome sequencing

Since $M E C P 2$ duplication in $\mathrm{I} 2$ might be on the active X-chromosome, we hypothesized the presence of an additional more serious pathogenic mutation on the other X-chromosome that would be responsible for skewing. As Affymetrix CytoScan HD Array detected no other likely harmful copy number variations in I2 but not in II2. Then we performed exome sequencing on DNA for females I2 and II2, we screened the mutations of genes in the OMIM catalog and found some that existed in I2 but not in II2. There were 12 mutations on the X-chromosomes, including one nonsense mutation and 11 missense mutations. Only three genes (NROB1 ,DMD, and USP9X) were involved in diseases, but there was no evidence to show that the three missense gene mutations were pathogenic (data not shown).

4. For both II2 and I2, the heterozygous SNP rs144237473 (chrX:73846325) in XIST only expressed in the X-chromosome with Xq28 duplication, which indicates that the Xq28duplication chromosome was inactive

To identify variants that may cause primary skewing in I2, we perfomed RNA sequencing in the peripheral mononuclear blood cells (PMBCs), data quality summary of RNA sequencing was listed in Supp. Table S2. we identified eight SNPs near the XIST region in I2 and II2 by exome sequencing (Supp. Table S3). All SNPs were consistent in I2 and II2 and therefore not considered potential primary skewing causes in I2. We also quantified the degree of skewing of XCI using a metric based on theXIST ASE from paired RNA-seq and DNA-seq data. It showed that heterozygous alleles of rs144237473 (chrX:73846325) (G:0/A:288) were expressed in a homozygous condition in II2, which is maternal inheritance. Similarly, the allelic expressions of rs144237473 (G:0/A:291) in I2 were complete skewed (Supp. Table S3, Figure 4). We also verified the SNP in DNA and cDNA by Sanger sequencing (Figure 3A), which revealed that each female's inactive $\mathrm{X}$-chromosome was the same chromosome with Xq28 duplication.

5. Most XCI genes in the X-chromosome with Xq28 duplication of the two females were not transcriptionally expressed

$\mathrm{XCI}$ statuses of X-linked genes were defined as escape, variable escape, and inactive. XCI genes, such as MECP2 and IRAK1, were only expressed in the active X (Xa) chromosome. Escape XCI genes, located mostly in the pseudoautosomal region, were expressed both in Xa chromosomes and the inactive X (Xi) chromosome. Variable escape XCI genes were determined to be variable XCI statuses (Tukiainen et al., 2017).

We listed all SNPs information on the X-chromosome detected by both RNA sequencing and WES. For I2 and II2, in the non-pseudoautosomal region, where most SNPs of XCI genes in the X-chromosome with Xq28 duplication were no transcription, and there was a lower overall transcription for variable and escaping XCI genes (Supp. Table S4).

6. MECP2 and IRAK1 on the Xq28-duplicated alleles of the two females were not transcriptionally expressed by RNA sequencing

Then we focused on the Xq28-duplicated region (Supp. Table S5). All SNP expressions of XCI genes (MECP2, IRAK1, TMEM187, TKTL1 ) in II2 and I2 were in a homozygous condition, indicating complete skewed XCI in II2 and I2. In the Xq28-duplicated region, there are several informative SNPs, which can definite parental origin, such as MECP2(chrX:154030040), IRAK1 (chrX:154013378, chrX:154018741, chrX:154019032), TMEM187 (chrX:153982271, chrX:153982294, chrX:153982503, chrX:153982797) (Figure 4 and Supp. Table S5). DNA and cDNA genotypes of MECP2 (chrX:154030040) were also detected by PCR-sanger sequencing (Figure 3B). It showed that the alleles inheriting from the mother I2, which is with Xq28 duplication, were not transcriptionally expressed. Likewise, in the variable XCI genes HCFC1, NAA10 
and the escaping XCI gene RENBP , the duplicated alleles inherited from the mother I2 were in lower expression. As informative SNPs of XCI genes presented in Figure 4 and Supp. Table S5, we found that the XCI genes $M E C P 2$ and IRAK1 on the Xq28-duplicated alleles of II2 and I2 were in silencing transcription.

\section{MECP2 and IRAK1 genes spanning the Xq28 duplication were not upregulated by RNA- seq and RT-PCR}

There were transcriptions of genes spanning the Xq28-duplicated region in the peripheral mononuclear blood cells (PMBCs) by RNA-seq. Expression dosages of the MECP2 and IRAK1 genes showed no significant difference between II2 and I2 (Supp. Table S6). We also detected the mRNA of MECP2 and IRAK1 in the two females and three normal females by RT-PCR (Figure 5). The expression dosages of $M E C P 2$ and $I R A K 1$ in the Xq28-duplication females were not upregulated compared to those of the normal females.

\section{DISCUSSION}

Here we reported two interstitial Xq28-duplication (involving $M E C P 2$ ) females (daughter and mother) with an asymptomatic phenotype. By HUMARA and RP2 assays, the chromosome with Xq28 duplication was inactive in female II2 (daughter) but was active in female I2 (mother). There should have been a related clinical phenotype in I2, and upregulated MECP2 gene expression, but I2 was an asymptomatic carrier. While by combing RNA-seq and RT-PCR, we found thatXIST only expressed in the Xq28-duplication chromosomes for I2 and II2. It indicated that the Xq28-duplication chromosomes were inactive. We also found that the XCI genes $M E C P 2$ and IRAK1 on two females' Xq28-duplicated alleles were not transcriptionally expressed nor upregulated by RNA-seq and qPCR. This can explain why the two females, especially I2, were asymptomatic carriers.

We summarized duplicated regions in Xq28, XCI states and the clinical features of the two asymptomatic females in this study and other seven symptomatic females, inheriting their duplication from their symptomatic mothers (Table 1) (Bijlsma et al., 2012; Novara et al., 2014; Reardon et al., 2010; Scott Schwoerer et al., 2014; Shimada et al., 2013). As similar with I2 in this study, only by HUMARA assay, some females' clinical phenotype did not necessarily correlate with the XCI pattern, such as the mothers of case 2, 3, 4, 6 and the daughter of case 7 . If XCI is really random, transcriptions of MECP2 on the duplicated allele and normal allele are random. Mothers of case 4, 6 with $M E C P 2$ duplication should have abnormal phenotypes, during to overexpression of MECP2, but they were asymptomatic. We thought it was because HUMARA assays could not always refect the real XCI states.

HUMARA/RP2 assays revealed methylation of the $\mathrm{X}$-chromosome at the genomic DNA level (Allen et al., 1992; Machado et al., 2014), but some reports asserted that the ratio of DNA methylation between alleles did not always reflect each allele's ratio of RNA expression. DNA methylation assays are not always representative of XCI (Swierczek et al., 2012). Clara Xiol et al. reported differences between the XCI pattern detected by HUMARA assay and final RNA levels of each $M E C P 2$ allele in Rett syndrome (RTT) patients caused by mutations in the MECP2 gene. This finding suggested that HUMARAassay did not directly determine the levels of MECP2, and there could be more factors than DNA methylation involved in the regulation of $M E C P 2$ transcript levels (Xiol et al., 2019). Ehrhart F. et al. also reported that there might be other factors involved in regulating $M E C P 2$ transcription and/or RNA degradation that would cause changes in the overall levels of functional MECP2 in RTT (Ehrhart et al., 2016). Therefore, we speculated that DNA methylation detected byHUMARA /RP2 assay might not necessarily be correlated with the severity of patients' clinical presentation with $M E C P 2$ gene defects.

$\mathrm{XCI}$ status is maintained by more than one factor. In a homozygous mouse, knockout of one of the XCI factors (XCIF) stanniocalcin 1 (STC1), was expected to have an XCI defect but was phenotypically normal. Remarkably, MECP2 was not overexpressed in female Stc1(-/-) mice (Bhatnagar et al., 2014), and animal experiment also confirmed that genetic reactivation of Xi-linked Mecp2 in cerebral cortical neurons of living mice can bear a homozygous XCIF deletion (Przanowski et al., 2018). It revealed the existence of a mechanism(s) that could compensate for a persistent XCI deficiency, so that X-linked gene MECP2expression is not upregulated. Meanwhile, many studies showed that duplication dosage inhibition of X-chromosome was 
also observed in 47,XXX females and many species (Meyer, 2005; Meyer, McDonel, Csankovszki, \& Ralston, 2004; Nielsen et al., 2020; L. Sun et al., 2013). These lower transcript levels suggested that a inhibition regulation at a higher level, for example, through mRNA degradation or other epigenetic mechanisms, such as histone modifications, maintaining the XCI status in addition to DNA methylation (Lee, 2011; Prestel, Feller, \& Becker, 2010). We speculated a potential inhibition mechanism might occurr at the transcriptional level in the unmethylated X-chromosome with $M E C P 2$ duplication, which resulted in a lack of up-regulation of duplicated $M E C P 2$ gene expressions in I2.

As a high-throughput RNA expression assay, RNA sequencing can measure the ratio of duplicated to normal alleles that have been activated directly for the MECP2 gene at the transcript level. Direct measurement of the allele expression may provide a better estimate of each inherited chromosome copy's true cellular activity, and it also increases our power to accurately estimate XCI, thus reflecting a greater influence of XCI on clinical manifestations.

The discovery of molecular mechanisms by DNA and RNA-seq in a patient's peripheral blood that may be correlated with phenotype in the central nervous system would provide potential benefits in clinical diagnostic cases that remain unresolved. But this finding is supported by some studies that have discovered a strong correlation in the gene expression profile of blood with the affected status of many neurological diseases, such as Parkinson's disease and Huntington's disease (Borovecki et al., 2005; Scherzer et al., 2007). Meanwhile, based on the analysis of ASE patterns by RNA-seq, the skewed XCI states of MECP2 were the same across 29 human tissues (Tukiainen et al., 2017), so we speculated that the allelic expression of MECP2 in the blood might reflect the state of the nervous system in Xq28-duplication patients.

In conclusion, we explained why transcriptions of $M E C P 2$ and $I R A K 1$ genes were not upregulated in the two Xq28-duplication females with opposite skewed XCI. We showed that XCI detected byHUMARA and RP2 assays did not always reflect the transcriptional level of Xq28 duplication. ASE assay by RNA sequencing, which reflects the transcription of the $M E C P 2$ alleles, is more directly correlated with the clinical phenotype. Meanwhile, we speculated there were other factors maintaining the XCI status in addition to DNA methylation, an additional inhibition mechanism might occur at the transcriptional level in the unmethylated $\mathrm{X}$-chromosome to counter balance the detrimental phenotype effects of MECP2duplication.

ACKNOWLEDGMENTS: We thank all the participants in the present study. We also thank Dr. Jiong Gao (BGI Genomics, BGI-Shenzhen, Shenzhen 518083, China) for his assistance in the preparation of this manuscript.

CONFLICT OF INTEREST: The authors have declared no conflicts of interest.

DATA AVAILABILITY STATEMENT: The data that support the findings of this study have been deposited in SRA database with the accession code PRJNA702822

FUNDING: This study was supported by the National Natural Science Foundation of China (Grant No. 81801441), the Key Research and Development Program of the Zhejiang Province (Grant No. 2019C03025), the Medical Health Science and Technology Project of Zhejiang Provincial Health Commission (Grant No. 2021KY772), the Public Welfare Technology Research Program of Zhejiang Province (Grant No. LGC20H200003).

AUTHOR CONTRIBUTIONS: MD designed the study. YL and YS performed FISH and SNP array. qPCR detection was carried out by YY1. RNA seq and WES were analyzed by YS, LW, and YH. MC and YY2 contributed the HUMARA and RP2 assays. YS and MD wrote the draft manuscript. All co-authors provided feedback on the estimates and contributed to the subsequent versions of the manuscript. All authors read and approved the final version of the manuscript.

\section{REFERENCES}

Allen, R. C., Zoghbi, H. Y., Moseley, A. B., Rosenblatt, H. M., \& Belmont, J. W. (1992). Methylation of HpaII and HhaI sites near the polymorphic CAG repeat in the human androgen-receptor gene correlates 
with X chromosome inactivation. Am J Hum Genet, 51(6), 1229-1239.

Bhatnagar, S., Zhu, X., Ou, J., Lin, L., Chamberlain, L., Zhu, L. J., . . . Green, M. R. (2014). Genetic and pharmacological reactivation of the mammalian inactive X chromosome. Proc Natl Acad Sci U S A, 111(35), 12591-12598. doi:10.1073/pnas.1413620111

Bijlsma, E. K., Collins, A., Papa, F. T., Tejada, M. I., Wheeler, P., Peeters, E. A., . . . Ruivenkamp, C. A. (2012). Xq28 duplications including MECP2 in five females: Expanding the phenotype to severe mental retardation. Eur J Med Genet, 55(6-7), 404-413. doi:10.1016/j.ejmg.2012.02.009

Borovecki, F., Lovrecic, L., Zhou, J., Jeong, H., Then, F., Rosas, H. D., . . . Krainc, D. (2005). Genomewide expression profiling of human blood reveals biomarkers for Huntington's disease. Proc Natl Acad Sci U S A, 102(31), 11023-11028. doi:10.1073/pnas.0504921102

Collins, A. L., Levenson, J. M., Vilaythong, A. P., Richman, R., Armstrong, D. L., Noebels, J. L., . . . Zoghbi, H. Y. (2004). Mild overexpression of MeCP2 causes a progressive neurological disorder in mice. Hum Mol Genet, 13(21), 2679-2689. doi:10.1093/hmg/ddh282

Ehrhart, F., Coort, S. L., Cirillo, E., Smeets, E., Evelo, C. T., \& Curfs, L. M. (2016). Rett syndrome - biological pathways leading from MECP2 to disorder phenotypes. Orphanet J Rare Dis, 11(1), 158. doi:10.1186/s13023-016-0545-5

El Chehadeh, S., Touraine, R., Prieur, F., Reardon, W., Bienvenu, T., Chantot-Bastaraud, S., . . . Faivre, L. (2017). Xq28 duplication including MECP2 in six unreported affected females: what can we learn for diagnosis and genetic counselling? Clin Genet, 91(4), 576-588. doi:10.1111/cge.12898

Fieremans, N., Bauters, M., Belet, S., Verbeeck, J., Jansen, A. C., Seneca, S., . . . Froyen, G. (2014). De novo MECP2 duplications in two females with intellectual disability and unfavorable complete skewed X-inactivation. Hum Genet, 133(11), 1359-1367. doi:10.1007/s00439-014-1469-6

Horga, A., Woodward, C. E., Mills, A., Parees, I., Hargreaves, I. P., Brown, R. M., . . . Hanna, M. G. (2019). Differential phenotypic expression of a novel PDHA1 mutation in a female monozygotic twin pair. Hum Genet, 138(11-12), 1313-1322. doi:10.1007/s00439-019-02075-9

Lee, J. T. (2011). Gracefully ageing at 50, X-chromosome inactivation becomes a paradigm for RNA and chromatin control. Nat Rev Mol Cell Biol, 12(12), 815-826. doi:10.1038/nrm3231

Machado, F. B., Machado, F. B., Faria, M. A., Lovatel, V. L., Alves da Silva, A. F., Radic, C. P., . . . MedinaAcosta, E. (2014). 5meCpG epigenetic marks neighboring a primate-conserved core promoter short tandem repeat indicate X-chromosome inactivation. PLoS One, 9(7), e103714. doi:10.1371/journal.pone.0103714

Makrythanasis, P., Moix, I., Gimelli, S., Fluss, J., Aliferis, K., Antonarakis, S. E., . . . Bottani, A. (2010). De novo duplication of MECP2 in a girl with mental retardation and no obvious dysmorphic features. Clin Genet, 78(2), 175-180. doi:10.1111/j.1399-0004.2010.01371.x

Meyer, B. J. (2005). X-Chromosome dosage compensation. WormBook, 1-14. doi:10.1895/wormbook.1.8.1

Meyer, B. J., McDonel, P., Csankovszki, G., \& Ralston, E. (2004). Sex and X-chromosome-wide repression in Caenorhabditis elegans. Cold Spring Harb Symp Quant Biol, 69, 71-79. doi:10.1101/sqb.2004.69.71

Nielsen, M. M., Trolle, C., Vang, S., Hornshoj, H., Skakkebaek, A., Hedegaard, J., . . . Gravholt, C. H. (2020). Epigenetic and transcriptomic consequences of excess X-chromosome material in 47,XXX syndromeA comparison with Turner syndrome and 46,XX females. Am J Med Genet C Semin Med Genet, 184(2), 279-293. doi:10.1002/ajmg.c.31799

Novara, F., Simonati, A., Sicca, F., Battini, R., Fiori, S., Contaldo, A., . . . Ciccone, R. (2014). MECP2 duplication phenotype in symptomatic females: report of three further cases. Mol Cytogenet, $7(1)$, 10 . doi:10.1186/1755-8166-7-10 
Orstavik, K. H. (2009). X chromosome inactivation in clinical practice. Hum Genet, 126(3), 363-373. doi:10.1007/s00439-009-0670-5

Prestel, M., Feller, C., \& Becker, P. B. (2010). Dosage compensation and the global re-balancing of aneuploid genomes. Genome Biol, 11(8), 216. doi:10.1186/gb-2010-11-8-216

Przanowski, P., Wasko, U., Zheng, Z., Yu, J., Sherman, R., Zhu, L. J., . . . Bhatnagar, S. (2018). Pharmacological reactivation of inactive X-linked Mecp2 in cerebral cortical neurons of living mice. Proc Natl Acad Sci U S A, 115(31), 7991-7996. doi:10.1073/pnas.1803792115

Ramocki, M. B., Peters, S. U., Tavyev, Y. J., Zhang, F., Carvalho, C. M., Schaaf, C. P., . . . Zoghbi, H. Y. (2009). Autism and other neuropsychiatric symptoms are prevalent in individuals with MeCP2 duplication syndrome. Ann Neurol, 66(6), 771-782. doi:10.1002/ana.21715

Ramocki, M. B., \& Zoghbi, H. Y. (2008). Failure of neuronal homeostasis results in common neuropsychiatric phenotypes. Nature, 455(7215), 912-918. doi:10.1038/nature07457

Reardon, W., Donoghue, V., Murphy, A. M., King, M. D., Mayne, P. D., Horn, N., \& Birk Moller, L. (2010). Progressive cerebellar degenerative changes in the severe mental retardation syndrome caused by duplication of MECP2 and adjacent loci on Xq28. Eur J Pediatr, 169(8), 941-949. doi:10.1007/s00431-010-1144-4

Scherzer, C. R., Eklund, A. C., Morse, L. J., Liao, Z., Locascio, J. J., Fefer, D., . . . Gullans, S. R. (2007). Molecular markers of early Parkinson's disease based on gene expression in blood. Proc Natl Acad Sci U S A, 104(3), 955-960. doi:10.1073/pnas.0610204104

Scott Schwoerer, J., Laffin, J., Haun, J., Raca, G., Friez, M. J., \& Giampietro, P. F. (2014). MECP2 duplication: possible cause of severe phenotype in females. Am J Med Genet A, 164A(4), 1029-1034. doi:10.1002/ajmg.a.36380

Shimada, S., Okamoto, N., Ito, M., Arai, Y., Momosaki, K., Togawa, M., . . . Yamamoto, T. (2013). MECP2 duplication syndrome in both genders. Brain Dev, 35(5), 411-419. doi:10.1016/j.braindev.2012.07.010

Sun, L., Johnson, A. F., Donohue, R. C., Li, J., Cheng, J., \& Birchler, J. A. (2013). Dosage compensation and inverse effects in triple X metafemales of Drosophila. Proc Natl Acad Sci U S A, 110(18), 7383-7388. doi:10.1073/pnas.1305638110

Sun, Y., Luo, Y., Qian, Y., Chen, M., Wang, L., Li, H., . . . Dong, M. (2019). Heterozygous Deletion of the SHOX Gene Enhancer in two Females With Clinical Heterogeneity Associating With Skewed XCI and Escaping XCI. Front Genet, 10, 1086. doi:10.3389/fgene.2019.01086

Swierczek, S. I., Piterkova, L., Jelinek, J., Agarwal, N., Hammoud, S., Wilson, A., . . . Prchal, J. T. (2012). Methylation of AR locus does not always reflect X chromosome inactivation state. Blood, 119(13), e100-109. doi:10.1182/blood-2011-11-390351

Tukiainen, T., Villani, A. C., Yen, A., Rivas, M. A., Marshall, J. L., Satija, R., . . . MacArthur, D. G. (2017). Landscape of X chromosome inactivation across human tissues. Nature, 550(7675), 244-248. doi:10.1038/nature24265

Van Esch, H. (1993). MECP2 Duplication Syndrome. In M. P. Adam, H. H. Ardinger, R. A. Pagon, S. E. Wallace, L. J. H. Bean, K. Stephens, \& A. Amemiya (Eds.), GeneReviews((R)). Seattle (WA).

Van Esch, H., Bauters, M., Ignatius, J., Jansen, M., Raynaud, M., Hollanders, K., . . . Froyen, G. (2005). Duplication of the MECP2 region is a frequent cause of severe mental retardation and progressive neurological symptoms in males. Am J Hum Genet, 77(3), 442-453. doi:10.1086/444549

Xiol, C., Vidal, S., Pascual-Alonso, A., Blasco, L., Brandi, N., Pacheco, P., . . . Rett Working, G. (2019). $\mathrm{X}$ chromosome inactivation does not necessarily determine the severity of the phenotype in Rett syndrome patients. Sci Rep, 9(1), 11983. doi:10.1038/s41598-019-48385-w 
Zito, A., Davies, M. N., Tsai, P. C., Roberts, S., Andres-Ejarque, R., Nardone, S., . . . Small, K. S. (2019). Heritability of skewed X-inactivation in female twins is tissue-specific and associated with age. Nat Commun, 10(1), 5339. doi:10.1038/s41467-019-13340-w

\section{Legends}

FIGURE 1. Xq28 intrachromosomal duplications were identified in female I2 (daughter) and II2 (mother) by SNP array, qPCR, and FISH analyses. (A) Pedigree of the family. (B) Blue bars indicate a $437 \mathrm{~kb}$ duplication in Xq28 (chrX:153858452-154332213 in the genome build GRCh38) and a $284 \mathrm{~kb}$ duplication in Xq27.3 (chrX:144988272-145271978 in the genome build GRCh38) in I2 and II2. (C) The relative ratio of the Xq28 (MECP2 and IRAK1 ) regions in I2 and II2 by qPCR. (D) For I2 and II2, a FISH experiment showed that the MECP2-containing probe (detected by probe RP11-119A22, Spectrum red) occurred only at Xq28, the X-chromosome centromere was labeled by probe Vysis CEP X (DXZ1) in the green spectrum. It demonstrated that the $\mathrm{Xq} 28$ duplications are tandem repeats that had not integrated elsewhere in the genome.

FIGURE 2. Polymorphic repeats for $H U M A R A / R P 2$ detected X-chromosome inactivation (XCI) pattern analyses. (A, D) A peak of 267 bp for HUMARA (381 bp for RP2 ) was observed by assaying the undigested PCR product of I1, and no peaks were observed for the HpaII digested product. (B, E) The undigested PCR product of I2 gave two peaks of 281 and $287 \mathrm{bp}$ for HUMARA (359 and $374 \mathrm{bp}$ forRP2 ), each, while only one peak of $281 \mathrm{bp}$ for HUMARA (374 bp for RP2 ) was observed with the HpaII digested product. I2 exhibited complete skewing of XCI, and the inactivated X-chromosome was linked with the 281 bp peak of HUMARA (374 bp peak of the RP2 ) PCR products. (C, F) The undigested PCR product of the proband II2 gave two peaks of 267 and $287 \mathrm{bp}$ for HUMARA (359 and $381 \mathrm{bp}$ for RP2 ). One X-chromosome linked with the $287 \mathrm{bp}$ for HUMARA (359 bp for RP2) was inhibited from the mother I2, and the other was inhibited from the father I1. The product of HpaII digestion gave only one peak of $287 \mathrm{bp}$ for HUMARA (359 bp for $R P 2$ ) to II2, which also demonstrated complete skewing of XCI. Still, the inactivated X-chromosome was linked with the $287 \mathrm{bp}$ for HUMARA (359 bp for RP2 ), which is different from that of the mother I2.

FIGURE 3. Sequence analysis of the genomic DNA and RT-PCR product of the XIST (chrX:73846325) and MECP2(chrX:154030040) cDNA from PMBCs. (A) The genotypes of XIST(chrX:73846325) were wild type, G>A Het, G>A Het, G>A Hom, G>A Hom in I:1 (DNA), I:2 (DNA), II:2 (DNA), I:2 (cDNA), II:2 (cDNA). (B) The genotypes of MECP2(chrX:154030040) were G>A Hom, wild type, G > A Het, wild type, G>A Hom, in I:1 (DNA), I:2 (DNA), II:2 (DNA), I:2 (cDNA), II:2 (cDNA). Yellow arrows indicate the SNPs.

FIGURE 4. XCI pattern and linkage analyses based on the polymorphic repeats for $H U M A R A / R P 2$ and RNA-seq. Schematic diagram of Xq28 duplication, HUMARA/RP2 PCR products, and RNA-seq in the pedigree. By HUMARA/RP2 assays, Xq28 duplication of I2 occurred in the X-chromosome, whose allele was linked with the 287 bp peak of HUMARA PCR product and 359 bp peak of the RP2 PCR product. The $\mathrm{X}$-chromosome was activated and delivered to the II2, but the X-chromosome of I2 was inactivated. Based on RNA-seq assays, for both I2 and II2, the heterozygous SNP rs144237473 (chrX:73846325) in XISTexpressed only in the X-chromosome with Xq28 duplication, and XCI genes MECP2 and IRAK1 expressions on the Xq28 duplicated alleles of the two females were not transcriptional expressions. Variable XCI genes $H C F C 1$ , NAA10 and the escaping XCI gene RENBP , the duplicated alleles inherited from the mother I2 were in lower expression. RNA-seq indicated that the duplicated Xq28 chromosome was inactive.

* Reported XCI status refers to the XCI status in the list available in Tukiainenet al. (2017)

\# It indicated that the X-chromosome was inactive based on the locus detected by DNA methylation assay or RNA-seq.

GT: genotype, the genotype of the locus.

() Allele depth; the number of reads supporting either the reference genotype or SNP genotype by RNA-seq. 
FIGURE 5. Relative expression fold of $M E C P 2$ (A) andIRAK1 (B) from peripheral blood mononuclear cells (PMBCs) in control females, I2, and II2 by qPCR. Expression dosages of theMECP2 and IRAK1 genes showed no significant difference in I2, II2, and control females.

\section{Hosted file}

table 1.pdf available at https://authorea.com/users/405865/articles/516705-lack-of-mecp2gene-transcription-on-the-duplicated-alleles-of-two-mecp2-duplication-females-withopposite-skewed-x-chromosome-inactivation

II1

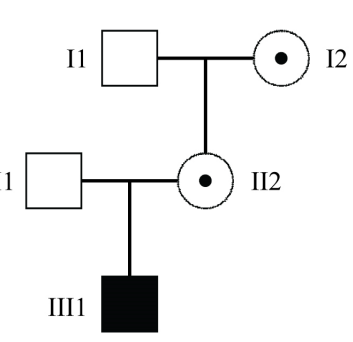

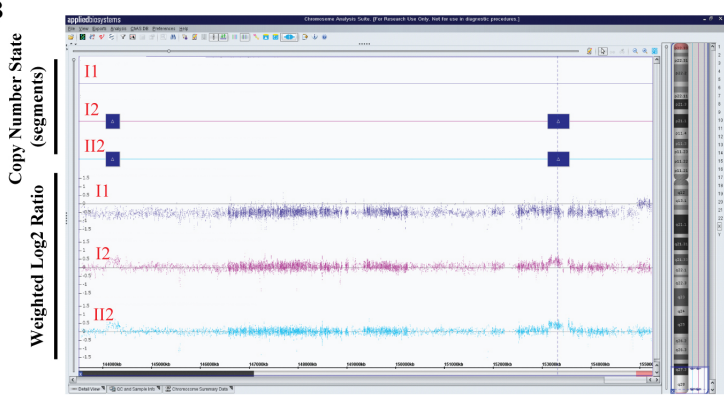

C

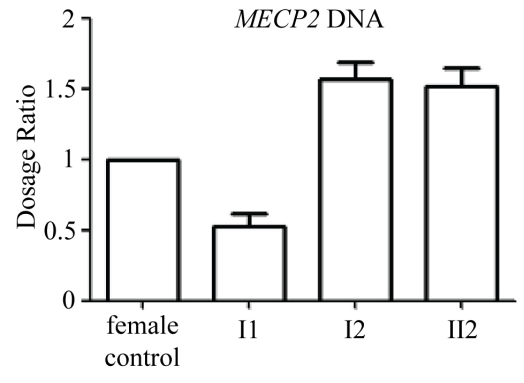

$\mathbf{E}$

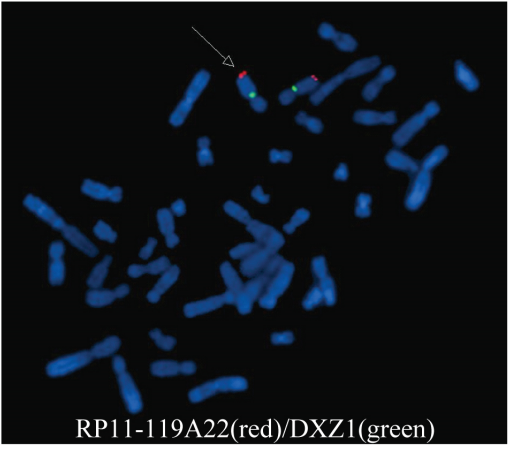

D

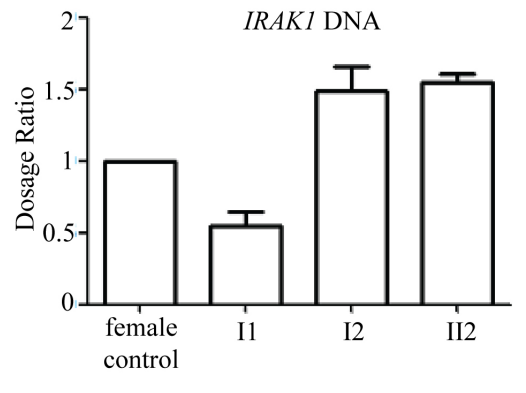

F

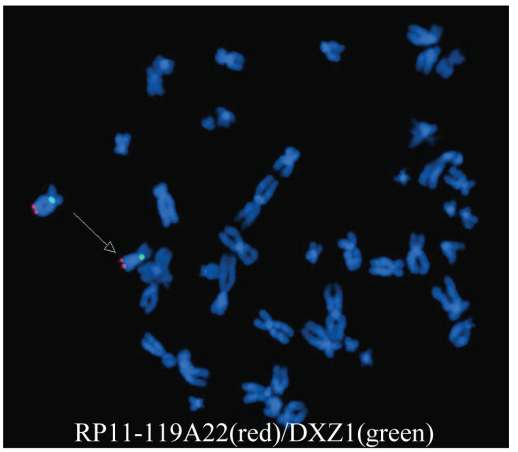



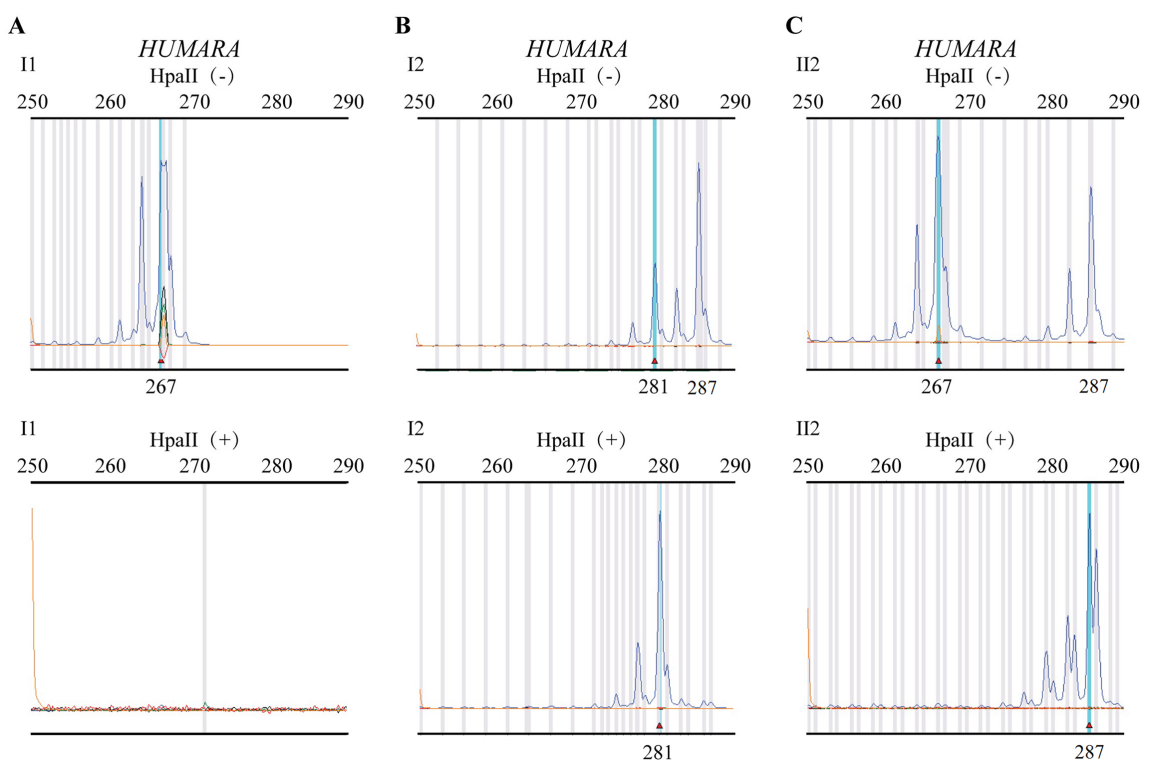

D

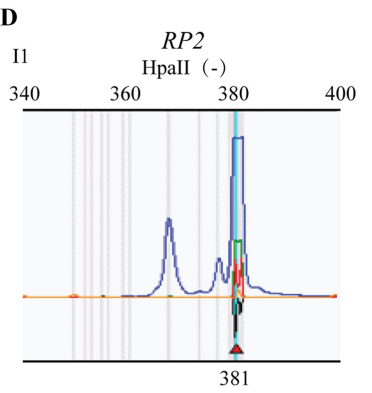

E

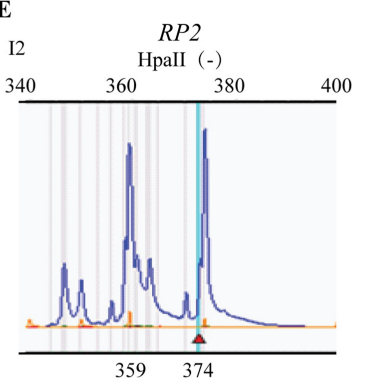

F

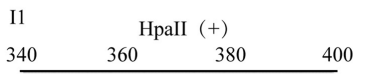

I2

II2
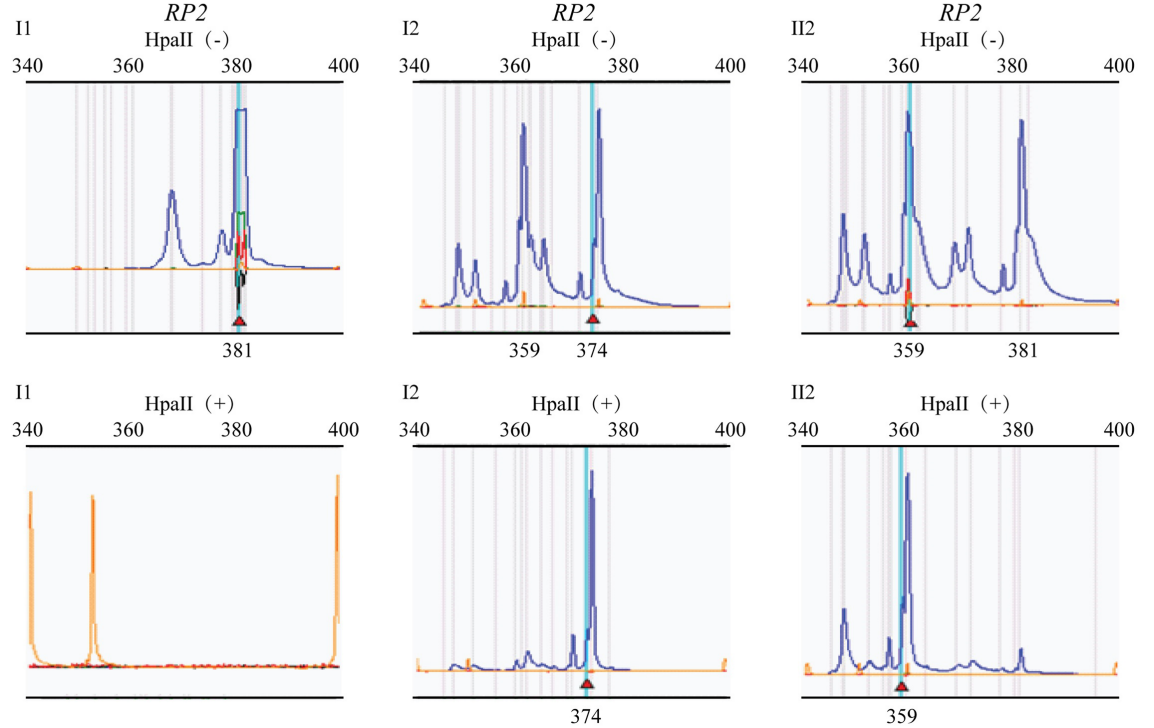

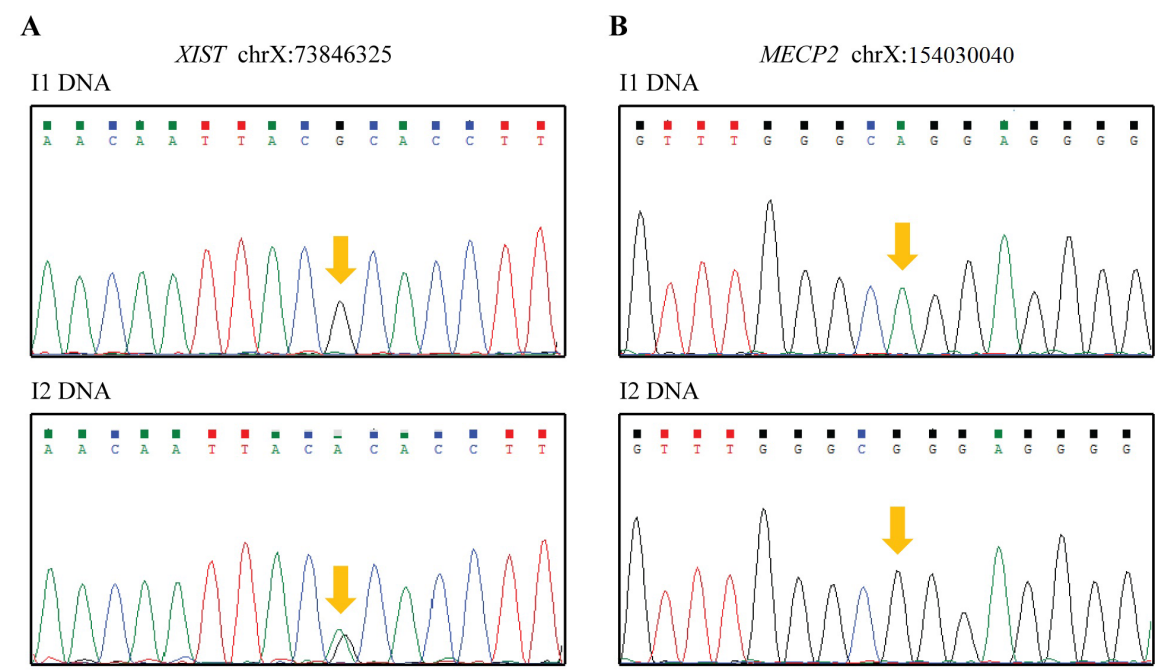

II2 DNA
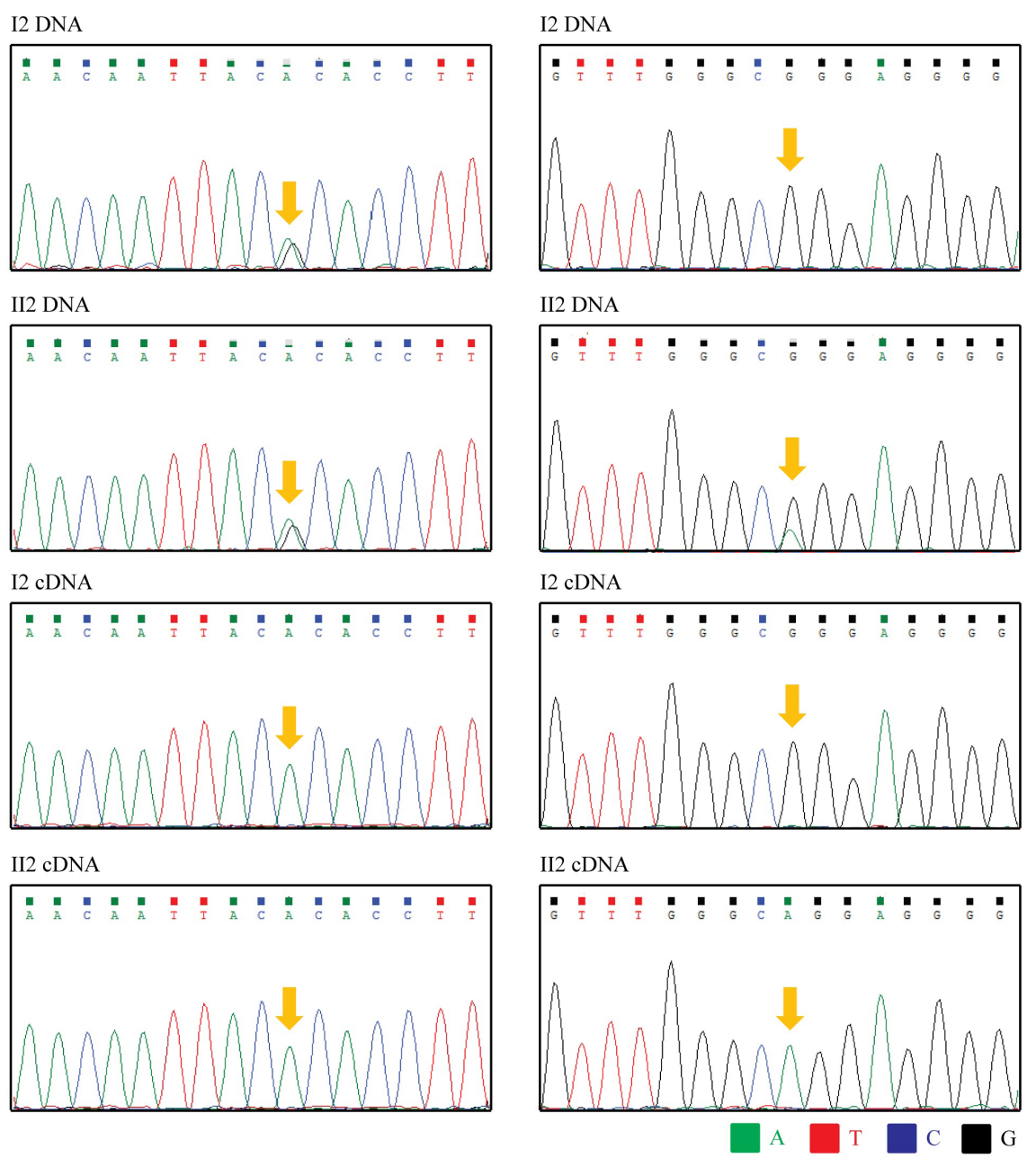

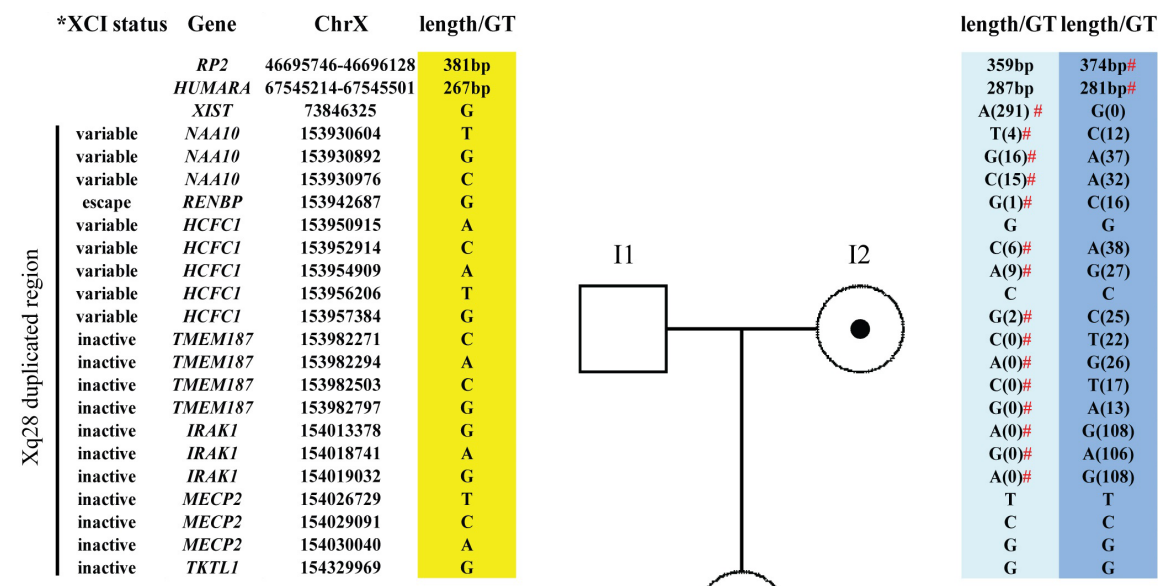

\begin{tabular}{|c|c|c|c|c|}
\hline \multirow[t]{4}{*}{ *XCI status } & Gene & ChrX & \multicolumn{2}{|c|}{ length/GT length/GT } \\
\hline & $R P 2$ & $46695746-46696128$ & 381 bp & 359 bp\# \\
\hline & HUMARA & 67545214-67545501 & $267 \mathrm{bp}$ & 287bp\# \\
\hline & XIST & 73846325 & G(0) & $\mathbf{A}(288) \#$ \\
\hline variable & NAA1O & 153930604 & $\mathrm{~T}$ & $\mathrm{~T}$ \\
\hline variable & NAA10 & 153930892 & G & G \\
\hline variable & NAA10 & 153930976 & $\mathrm{C}$ & $\mathrm{C}$ \\
\hline escape & RENBP & 153942687 & G & G \\
\hline variable & HCFC1 & 153950915 & $A(67)$ & $\mathbf{G}(9) \#$ \\
\hline variable & HCFCl & 153952914 & C & C \\
\hline variable & HCFC1 & 153954909 & A & A \\
\hline variable & HCFCl & 153956206 & $\mathrm{~T}(\mathbf{8 3})$ & C(8)\# \\
\hline variable & HCFCl & 153957384 & G & G \\
\hline inactive & TMEM187 & 153982271 & $\mathrm{C}$ & $\mathrm{C}$ \\
\hline inactive & TMEM187 & 153982294 & A & $\mathbf{A}$ \\
\hline inactive & TMEM187 & 153982503 & $\mathrm{C}$ & $\mathrm{C}$ \\
\hline inactive & TMEM187 & 153982797 & G & G \\
\hline inactive & IRAKI & 154013378 & $G(147)$ & $\mathbf{A}(\mathbf{0}) \#$ \\
\hline inactive & IRAKI & 154018741 & $A(25)$ & G(0)\# \\
\hline inactive & IRAKI & 154019032 & $G(192)$ & $\mathbf{A}(\mathbf{0}) \#$ \\
\hline inactive & MECP2 & 154026729 & $T$ & $\mathrm{~T}$ \\
\hline inactive & MECP2 & 154029091 & C & $\mathrm{C}$ \\
\hline inactive & МECP2 & 154030040 & A(97) & $\mathbf{G}(\mathbf{0}) \#$ \\
\hline inactive & TKTL1 & 154329969 & & \\
\hline
\end{tabular}

A

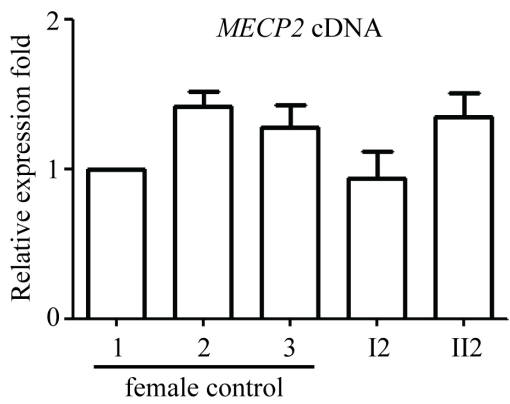

B

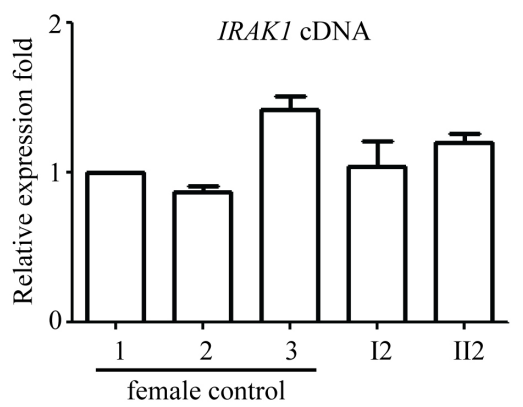

